\title{
External stimuli response on a novel chitosan hydrogel crosslinked with formaldehyde
}

\author{
A SINGH, S S NARVI, P K DUTTA and N D PANDEY* \\ Department of Chemistry, Motilal Nehru National Institute of Technology, Allahabad 211 004, India
}

MS received 2 May 2005

\begin{abstract}
Keeping in mind the significance of hydrogels as an external stimuli sensitive super absorbing material, some transparent covalent hydrogels of chitosan were prepared by crosslinking with varying amounts of formaldehyde solution used as crosslinking agent. The characteristics of hydrogels were investigated by Fourier transform infrared (FT-IR) spectroscopy and swelling experiments. The effect of crosslinking agent on water absorbency has been investigated. The hydrogels exhibited a relatively higher swelling ratio in the range of 2066-3306\% and equilibrium water content (EWC) in the range of $95 \cdot 38-97 \cdot 06 \%$ at $\mathrm{pH} 7$ and $35^{\circ} \mathrm{C}$ temperature. The influence of external stimuli such as $\mathrm{pH}$, temperature, and ionic strength of the swelling media on equilibrium swelling properties has been observed. Hydrogels showed a typical pH and temperature responsive behaviour such as low $\mathrm{pH}$ and high temperature has maximum swelling while high $\mathrm{pH}$ and low temperature show minimum swelling. An increase in the ionic strength of swelling media caused a continuous decrease in the swelling of hydrogels at both acidic and basic $\mathrm{pH}$.
\end{abstract}

Keywords. Chitosan; hydrogel; crosslinking; formaldehyde; swelling.

\section{Introduction}

These days biocompatible and biodegradable hydrogels find a wide application in the field of medicine, pharmaceutics, tissue engineering, chromatography, metal chelation etc. This has prompted scientists and technologists to give much emphasis to develop some effective, useful and bio-degradable hydrogels from a sustainable resource (Guan et al 1996; Oliverira and Glasser 1996; Pascual et al 1996; Rathna et al 1996).

Hydrogels are three dimensional hydrophilic polymeric networks which absorb and retain 10-20\% (an arbitrary lower limit) up to thousands of time of water or biological fluid than their dry weights. Hydrogels may be chemically stable or they may degrade and eventually disintegrate and dissolve (Hoffman 2002). To avoid this dissolution/degradation, a controlled crosslinking is introduced within the hydrogels. Stimuli responsive hydrogels which swell and contract in response to external condition have been explored (Qui and Park 2001; Hennink and Nostrum 2002). They have been considered to be useful in bioseparation and medical applications. Many structural factors (e.g. charge, concentration and $\mathrm{pKa}$ of the ionizable group, degree of ionization, crosslink density and hydrophilicity) influence the degree of swelling of ionic polymers (Askadskii 1990; Lee et al 1999; Wu et al 2001). In

*Author for correspondence (pandey_nd@ rediffmail.com) addition, properties of the swelling medium (e.g. $\mathrm{pH}$, ionic strength and the counter ion and its valency) affect the swelling characteristics (Castel et al 1990; Lee and Lin 2001; Gupta et al 2002).

In recent years, much attention has been given on the development of hydrogels from natural, biodegradable and biocompatible polymeric materials. Among other biodegradable polymers, chitosan, a linear polysaccharide has been found to be a good chemical entity for synthesizing hydrogels because of its greater crosslinking ability due to the presence of amino $\left(-\mathrm{NH}_{2}\right)$ group (Ravi Kumar 2000). The biodegradability, biocompatibility and other unique properties of chitosan have been used in a variety of areas such as medicine, pharmaceutics (Kim et al 1992; Gong et al 2000; Illum et al 2000; Miao et al 2001), tissue engineering (Drury and Mooney 2003), antimicrobial agents (Zhao et al 2003), textiles, chromatography (Bayramoglu and Arica 2002), metal chelation (Denzili et al 2000), and other industrial as well as biomedical applications.

A number of methods have been used for the crosslinking of chitosan, such as chemical crosslinking with glutaraldehyde (Hirano et al 1977), blocked diisocyanate (LinGibson et al 2003) and with Mo(IV) polyoxyanions (Draget et al 1992). Chemical crosslinking method seems to be a better approach to improve the wet strength of hydrogels. The polymers with hydroxyl group require drastic conditions (low $\mathrm{pH}$, high temperature, etc) in order to establish crosslinking with aldehydes, whereas amine con- 
taining polymers can be crosslinked with the same reagent under mild conditions where Schiff bases are formed. A few procedures such as semi and full-IPN hydrogels of chitosan/poly ( $\mathrm{N}$-isopropyl acrylamide) (Wang et al 2000, 2001) have been published in which formaldehyde was used as crosslinking agent for production of hydrogels. A blend membrane of chitosan and poly (vinyl alcohol) was prepared and treated with formaldehyde (Yang et al 2004). The thermal stability of membrane was enhanced by formaldehyde as crosslinking agent. The super water reservoir gel by deacetylated chitin i.e. chitosan, crosslinked with formaldehyde solution was also prepared (Lin et al 2003); but they used EDTA in place of $\mathrm{HCl}$ for demineralization, in preparation of chitin. The molecular weight of chitin obtained from this process is much higher than those obtained from $\mathrm{HCl}$ process. We further promote their work and observe the influence of external stimuli on swelling behaviour of these hydrogels.

The present study reports the preparation of blend of chitosan hydrogels crosslinked with formaldehyde. The spectroscopic (FTIR) characterization of hydrogel, effect of extent of crosslinking and effect of temperature, $\mathrm{pH}$ and ionic strength of swelling media on swelling behaviour of hydrogels were observed. The formaldehyde crosslinked chitosan hydrogels exhibit far better swelling behaviour than the other reported chitosan hydrogels.

\section{Experimental}

\subsection{Materials}

Chitosan with an average molecular weight of 1,00,000 and degree of deacetylation, $79.1 \%$, was supplied by Central Institute of Fisheries Technology (CIFT), Cochin and was used without further purification. Glacial acetic acid (Qualikems Fine Chemicals, India), formaldehyde solution (Thomas Baker, India) and other chemicals used were of AR grade.

\subsection{Preparation of chitosan hydrogels}

The synthesis of hydrogels was carried out by crosslinking of the chitosan with formaldehyde as per reported method (Lin et al 2003). In short, 5 samples of $0 \cdot 1 \mathrm{~g}$ chitosan each were dissolved in $7 \mathrm{ml} 1 \%$ glacial acetic acid solutions and after adding varying amounts of $5 \mathrm{~mol} / \mathrm{L}$ formaldehyde solution (crosslinking agent) into each sample, water was added till the mass of the concentrated gel became $12 \cdot 1 \mathrm{~g}$. The amount of formaldehyde solution into each sample is listed in table 1.

The crosslinking time of gel formation could be varied between 5 and $120 \mathrm{~min}$ and gel will be transparent after crosslinking.
Table 1. Composition of hydrogels.

\begin{tabular}{|c|c|c|c|}
\hline $\begin{array}{l}\text { Sample } \\
\text { no. }\end{array}$ & & & $\begin{array}{c}\text { Formaldehyde } \\
\text { solution (ml) }\end{array}$ \\
\hline 1 & $0 \cdot 1$ g Chitosan & $7 \mathrm{ml} \mathrm{1 \%} \mathrm{Glacial}$ & $1 \cdot 5$ \\
\hline 2 & & acetic acid & $2 \cdot 5$ \\
\hline 3 & & & $3 \cdot 5$ \\
\hline 4 & & & $4 \cdot 5$ \\
\hline 5 & & & $5 \cdot 5$ \\
\hline
\end{tabular}

\subsection{IR spectral analysis}

The Fourier transform infrared (FT-IR) transmission spectra were obtained from the film sample on a Perkin Elmer Spectrum RX1 FT-IR spectrometer.

\subsection{Swelling measurements}

After completion of crosslinking reaction, a weighed amount of hydrogel of each sample was immersed in solutions of desired $\mathrm{pH}(2-10)$, ionic strength $(I=0.01-$ $0 \cdot 2 \mathrm{M}$ ) and temperature, $35^{\circ} \mathrm{C}$, until they swelled to equilibrium. The $\mathrm{pH}$ values were precisely checked by a pH-meter (Decibel DP510) previously standardized with buffer solutions of $\mathrm{pH} 4$ and 9·2. The fully swollen samples were weighed after the removal of excessive surface water with filter paper.

The swelling ratio can be calculated from the equation

$$
\text { Swelling ratio }(\%)=\left[\left(W_{\mathrm{s}}-W_{\mathrm{d}}\right) / W_{\mathrm{d}}\right] \times 100,
$$

where $W_{\mathrm{s}}$ represents the weight in the equilibrium swollen state of the sample at experimental temperature and $W_{\mathrm{d}}$ the weight of the dry state of the sample.

Equilibrium water content (EWC) was calculated from the equation

$$
\operatorname{EWC}(\%)=\left[\left(W_{\mathrm{e}}-W_{\mathrm{d}}\right) / W_{\mathrm{e}}\right] \times 100,
$$

where $W_{\mathrm{e}}$ is the weight in the equilibrium swollen state of the sample.

\section{Results and discussion}

There are various kinds of crosslinking agents that can crosslink chitosan. Among them aldehydes and anhydrides e.g. formaldehyde, acetaldehyde, glutaraldehyde, acetic anhydride etc are commonly used as crosslinking agents because they are cheap and easily available. The crosslinking capacity, hydrophilic properties and water retention efficiency of hydrogel depends on the crosslinking agent. The polymers with hydroxyl group require drastic conditions (low $\mathrm{pH}$, high temperature, etc) in order to establish crosslinking with aldehydes whereas amine containing polymers can be crosslinked with the same re- 
agent under mild conditions where Schiff bases are formed.

\subsection{Mechanism of crosslinking reaction}

The chitosan is easily crosslinked with formaldehyde by Schiff base mechanism. The mechanism of crosslinking reaction is given in figure 1 .

\subsection{IR spectral analysis}

The FT-IR spectra of chitosan and chitosan hydrogel are shown in figure 2 . The spectra of hydrogel is similar to those of the original chitosan. The characteristic peaks of chitosan are located at $3429 \mathrm{~cm}^{-1}$ for the hydroxyl group and $1592 \mathrm{~cm}^{-1}$ for the amino group (Qu et al 2000). A peak at $1656 \mathrm{~cm}^{-1}$ is due to carbonyl stretching vibration of remaining acetamide group in chitosan ( $\mathrm{Qu}$ et al 2000). Figure 2(b) shows a significant peak at $1566 \cdot 8 \mathrm{~cm}^{-1}$ due to imine bonds $(\mathrm{C}=\mathrm{N})$ formed by crosslinking reaction between amino groups in the chitosan and aldehyde group

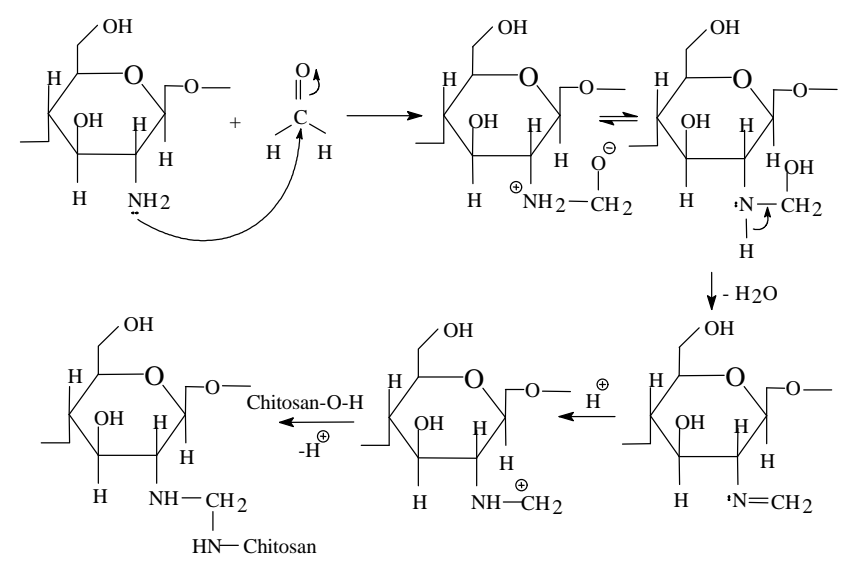

Figure 1. Crosslinking reaction of chitosan with formaldehyde.

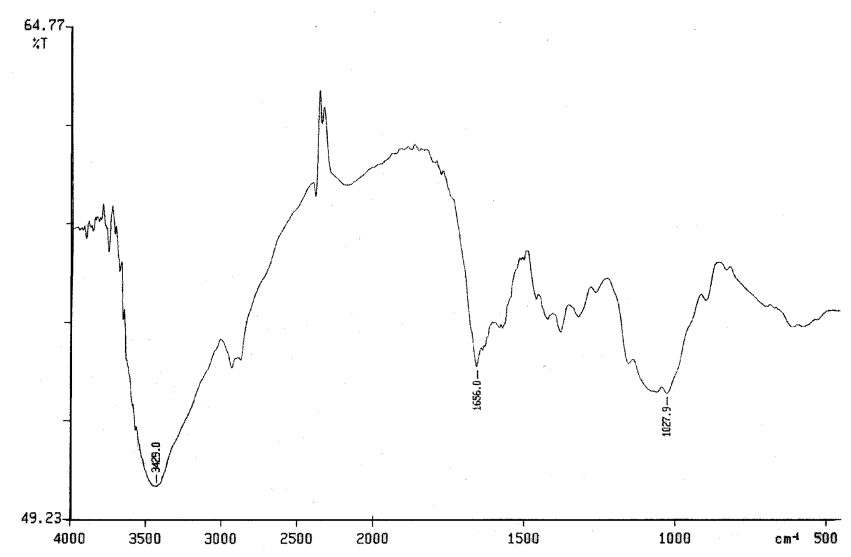

(a) in formaldehyde (Lee et al 2000). This characteristic peak confirms the formation of Schiff base after the reaction of formaldehyde with chitosan. The peak of the ether group in figure 2(a) becomes stronger and shifted slightly from $1059-1070 \cdot 5 \mathrm{~cm}^{-1}$ in figure 2(b), suggesting the formation of a new open chain ether linkage in hydrogel after crosslinking reaction.

\subsection{Swelling behaviour of hydrogels}

Chitosan hydrogels are ionized hydrogels, therefore, their swelling behaviour depends on both characteristics of the chemical structure and the medium. Generally, the extent of swelling of hydrogel at equilibrium increases with (a) an increase in the concentration of functional ionizable groups in the network and (b) a decrease in the extent of crosslinking occurring during the synthesis process.

3.3a Effect of crosslinking agent on swelling: Swelling ratio and EWC of all 5 samples at $\mathrm{pH} 7$ and $35^{\circ} \mathrm{C}$ temperature are plotted in figures 3 and 4 . All hydrogels reached to equilibrium within $24 \mathrm{~h}$. Sample 3 had the highest swelling ratio and EWC while sample 1 had the least.

The strength and water preservation efficiency of hydrogel is greatly affected by the amount of crosslinking agent. The linear structure of chitosan molecule can be transformed into network structure through crosslinking and water molecule can be preserved in this structure. As shown in figures 3 and 4 the swelling ratio and EWC of samples 1 and 2 was low because when the amount of crosslinking agent was low, the extent of crosslinking in the hydrogel was also low, which results in decrease in strength and water preservation efficiency of hydrogel. But in the case of samples 4 and 5, excessive amount of crosslinking agent was used, the degree of crosslinking was higher, resulting in the decrease of network volume for water preservation efficiency of the hydrogel. Similar results have been reported in literature (Wu et al 2001;

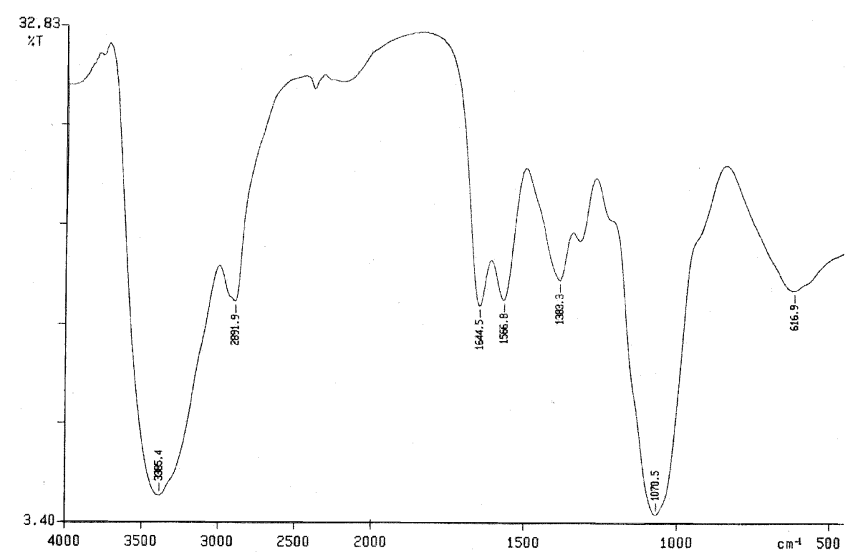

(b)

Figure 2. FT-IR spectra of (a) chitosan and (b) chitosan hydrogel crosslinked with formaldehyde. 
Lin-Gibson et al 2003). In case of sample 3, amount of crosslinking agent was neither low nor high; therefore, it had highest water preservation efficiency. In other words, sample 3 had highest swelling ratio and EWC. So it is clear from the observations and plots that the swelling ratio and EWC of hydrogels increased till a certain point with increasing amount of crosslinking agent, but decreased with excessive amount of crosslinking agent.

$3.3 \mathrm{~b} p H$ response characteristics: To investigate the swelling behaviour at various $\mathrm{pH}$ levels, the hydrogel samples were swollen in several buffer solutions of $\mathrm{pH} 2$, 4, 7, 9 and 10 at room temperature. Figure 5 shows the $\mathrm{pH}$ dependent swelling behaviours of fully swollen hydrogels. The hydrogels show a lower specific solution content at basic $\mathrm{pH}$ as compared with acidic $\mathrm{pH}$. Since high concentration of charged ionic groups in the hydrogel increases swelling due to osmosis and charge repulsion. Thus, swelling decreases when the degree of ionization of hydrogel bound groups decreases. At $\mathrm{pH} 2-4$, unreacted amino groups of chitosan ionized in an acid, the ammonium ion. The ammonium ion would be attached to the hydrogels by ionic bonds. Therefore, the weight of the

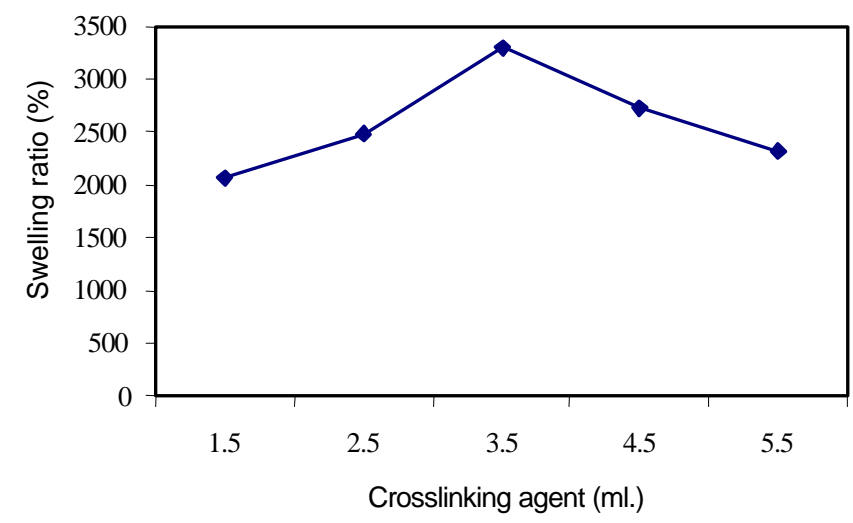

Figure 3. Swelling ratio (\%) of hydrogels with varying amounts of crosslinking agent.

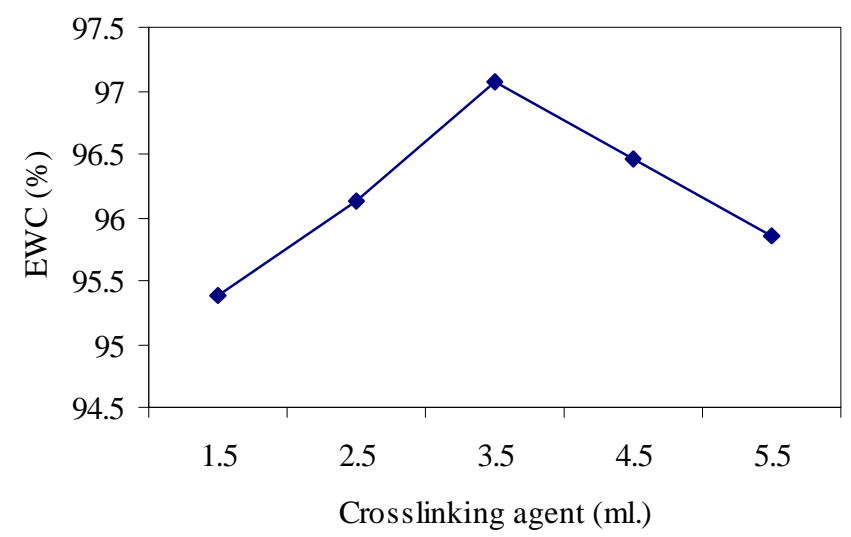

Figure 4. EWC $(\%)$ of hydrogels with varying amounts of crosslinking agent. hydrogels increased in acidic buffer. At high $\mathrm{pH}(\mathrm{pH} 7-10)$, however, amino group of chitosan is in the form of $-\mathrm{NH}_{2}$, resulting in lower EWC than at lower $\mathrm{pH}$. This $\mathrm{pH}$ sensitive behaviour is typical of ionic hydrogels.

Due to high dissociation of intermolecular ammonium salt, samples 1 and 2 were dissolved at $\mathrm{pH} 2$. Since, sample 3 possesses an appropriate amount of crosslinking agent, which results in neither high nor low extent of crosslinking in hydrogel structure. Thus, it shows highest swelling degree, with the highest total water content at all experimental conditions.

Samples 1 and 2 containing lesser amount of crosslinking agent, thus greater numbers of unreacted amino groups are available there for ionization in acidic $\mathrm{pH}$ and therefore, the EWC of these samples were slightly higher than samples 4 and 5 at $\mathrm{pH}$ 4. But due to low extent of crosslinking degree these samples had lower EWC than

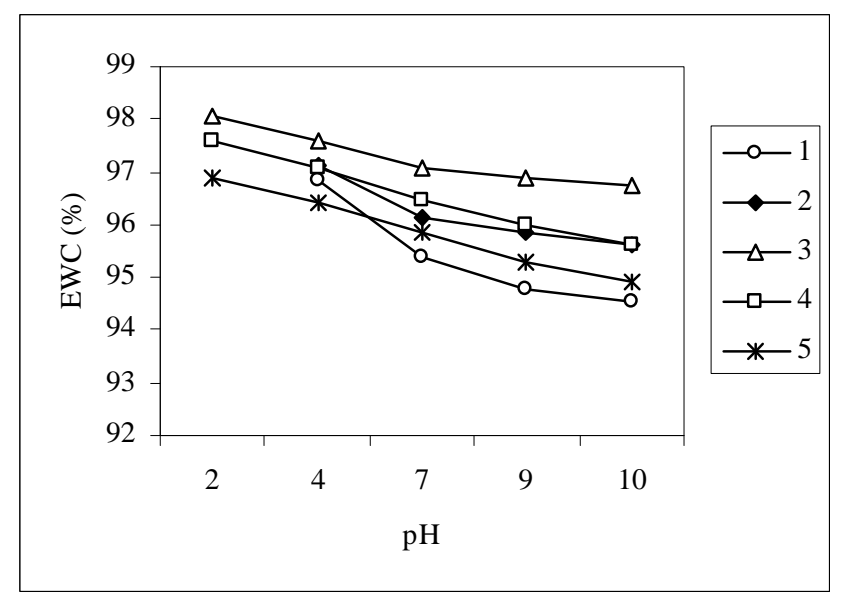

Figure 5. pH-dependent swelling behaviour of chitosan hydrogels.

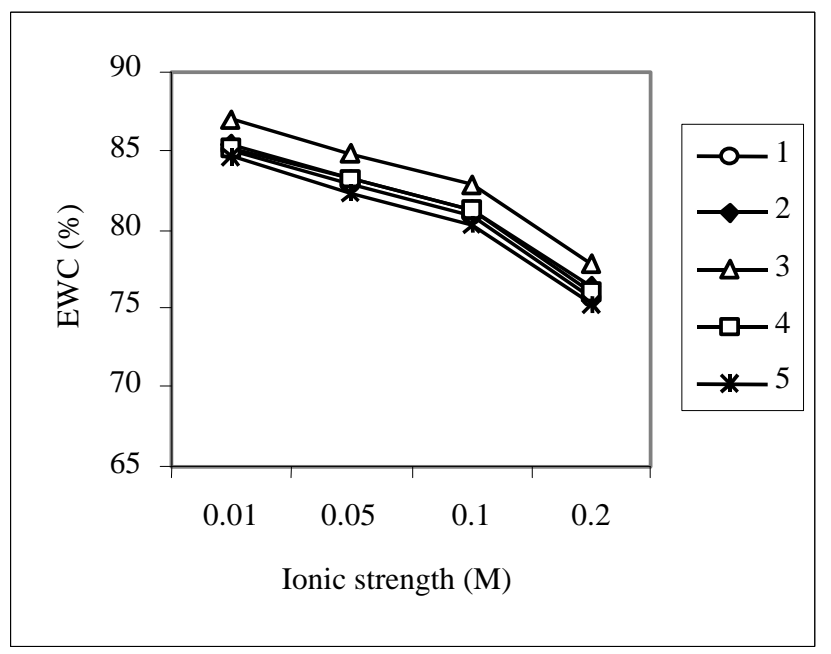

Figure 6. Effect of ionic strength on the equilibrium water content $(\%)$ of chitosan hydrogels at $\mathrm{pH} 3$. 
sample 3. Sample 5 possesses excessive amount of crosslinking agent, thus due to less available numbers of amino groups for ionization, it showed lowest EWC at acidic $\mathrm{pH}$. Therefore, on the basis of observations, it can be said that only greater number of unreacted amino groups are not necessary criteria for highest total water content; degree of crosslinking is also an essential parameter for highest total water content at different $\mathrm{pH}$ levels.

$3.3 \mathrm{c}$ Ionic strength responsive characteristics: The effect of ionic strength ( $\log \gamma \pm$ anions or cations) on the equilibrium water content of chitosan hydrogels at $35^{\circ} \mathrm{C}$ are shown in figures 6 and 7. To obtain maximum and minimum swelling of the hydrogels, the $\mathrm{pH}$ of the solution was fixed at 3 and 8 . Figures 6 and 7 clearly show that an increase in the ionic strength caused a continuous decrease in the swelling of hydrogels at both acidic and basic $\mathrm{pH}$. This is a well known phenomenon, commonly observed in ionic hydrogels. It is often attributed to a

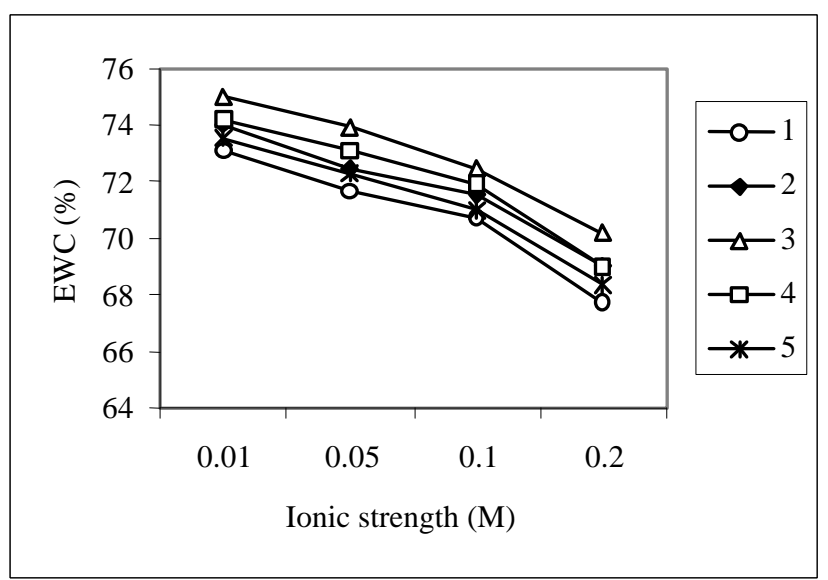

Figure 7. Effect of ionic strength on the equilibrium water content $(\%)$ of chitosan hydrogels at $\mathrm{pH} 8$.

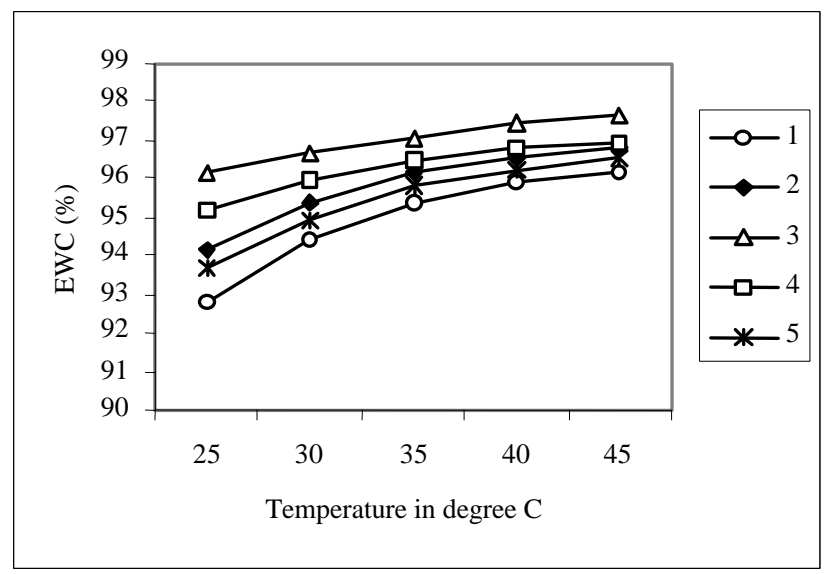

Figure 8. Effect of temperature on the equilibrium water content $(\%)$ of chitosan hydrogels at $\mathrm{pH} 7$. charge screening effect of the additional anions causing a non perfect cation-cation electrostatic repulsion, leading to a decreased osmotic pressure difference between the hydrogel network and the external solution and causing shrinkage of the gel. This effect becomes more pronounced in acidic condition ( $\mathrm{pH} 3)$.

3.3d Temperature responsive characteristics: Figure 8 represents the effect of temperature on the equilibrium water content of chitosan hydrogels in $\mathrm{pH} 7$ buffer solution at a temperature range from $25-45^{\circ} \mathrm{C}$. As the temperature of the hydrogel in the swelling state increased, the water content of the hydrogel also increased. All hydrogels show a temperature responsive swelling behaviour due to the association/dissociation of hydrogen bonding by the amino groups of chitosan within hydrogels. Therefore, EWC of all hydrogels increased with increasing temperature.

\section{Conclusions}

Chitosan hydrogels were synthesized by varying the amounts of formaldehyde solution used as a crosslinking agent. All hydrogels reached to equilibrium swelling within $24 \mathrm{~h}$. The swelling ratio and EWC of these hydrogels got affected by the amount of crosslinking agent. The swelling ratio and EWC of hydrogels increased till a certain point with increasing amount of crosslinking agent, but decreased with excessive amount of crosslinking agent. Sample 3 had the highest swelling ratio and EWC, while sample 1 had the least. These novel hydrogels with such a high swelling capacity could be used as superwater absorbent. The effect of external stimuli such as $\mathrm{pH}$, temperature, and ionic strength of the swelling media on equilibrium swelling properties has also been investigated. Hydrogels showed a typical $\mathrm{pH}$ and temperature responsive behaviour such as low $\mathrm{pH}$ and high temperature had maximum swelling while high $\mathrm{pH}$ and low temperature showed minimum swelling. An increase in the ionic strength of swelling media caused a continuous decrease in swelling of hydrogels at both acidic and basic $\mathrm{pH}$. Ionic repulsion between charge groups incorporated in the gel matrix by an external $\mathrm{pH}$ modulation could be assumed as the main driving force responsible for such abrupt swelling changes.

\section{References}

Askadskii A A 1990 Polym. Sci. USSR 322061

Bayramoglu G and Arica M Y 2002 Colloids and Surfaces A: Physicochem. \& Eng. Asp. 20041

Castel D, Richard A and Audebert R 1990 J. Appl. Polym. Sci. 3911

Denzili A, Yavuz H and Arica M Y 2000 Colloids Surf. A174 307 
Draget K I, Varum K M, Moen E, Gynnild H and Smidsrod O 1992 Biomaterials 13635

Drury J L and Mooney D J 2003 Biomaterials 244337

Gong H P, Zhong Y H, Li J C, Gong Y D, Zhao N M and Zhang X F 2000 J. Biomed. Mater. Res. 52285

Guan Y L, Shao L, Liu J and Yao K D 1996 J. Appl. Polym. Sci. 612325

Gupta P, Vermani K and Garg S 2002 Drug Discov. Today 7 569

Hennink W E and Nostrum C F van 2002 Adv. Drug Delivery Rev. 5413

Hirano S, Yamaguchi R, Matsuda N, Miura O and Kondo Y 1977 Agric. \& Biol. Chem. 411547

Hoffman A S 2002 Adv. Drug Delivery Rev. 433

Illum L, Watts P, Fisher A N, Gill I J and Davis S S 2000 STP Pharm. Sci. 1089

Kim J H, Kim J Y, Lee Y M and Kim K Y 1992 J. Appl. Polym. Sci. 441823

Lee J W, Kim S Y, Kim S S, Lee Y M and Lee K H 1999 J. Appl. Polym. Sci. $\mathbf{7 3} 113$

Lee S J, Kim S S and Lee Y M 2000 Carbohydrate Polym. 41 197

Lee W F and Lin G H 2001 J. Appl. Polym. Sci. 791665
Lin-Gibson S, Walls H J, Kennedy S B and Welsh E R 2003 Carbohydrate Polym. 54193

Lin Y, Yang D, He L-Z and Li S-D 2003 J. Polym. Mater. 20 417

Miao Y, Chia L S, Goh N K and Tan S N 2001 Electroanalysis 13347

Oliverira W D and Glasser W G 1996 J. Appl. Polym. Sci. 6181

Pascual B, Castellano I, Vazquez B, Gurruchaga M and Goni I 1996 Polymer 371005

Qui Y and Park K 2001 Adv. Drug Delivery Rev. 53321

Qu X, Wirsen A and Albertsson A-C 2000 Polymer 414589

Rathna G V N, Rao D V M and Chatterji P R 1996 JMS-Pure Appl. Chem. A33 1199

Ravi Kumar M N V 2000 React. \& Funct. Polym. 461

Wang M Z, Qiang J C, Fang Y, Hu D D, Cui Y L and Fu X G 2000 J. Polym. Sci. Part A: Polym. Chem. 38474

Wang M, Fang Y and Hu D 2001 React. \& Funct. Polym. 48 215

Wu J, Lin J, Wei C and Li G 2001 Polym. Int. 501050

Yang J M, Su W Y, Lu T L and Yang M C 2004 J. Membr. Sci. 23639

Zhao L, Mitomo H, Zhai M, Yushii F, Nagasawa N and Kume T 2003 Carbohydrate Polym. $\mathbf{5 3} 439$ 